\title{
Zebrafish models for transcriptional regulation in development and stem cell differentiation
}

\author{
Shannon Fisher ${ }^{1}$ \\ ${ }^{1}$ Institute of Genetic Medicine, and Department of Cell Biology, Johns Hopkins University School of Medicine, MD, USA
}

The availability of multiple vertebrate genome sequences has revealed extensive evolutionary conservation in non-coding sequence. We presume that many of these conserved non-coding sequences function in transcriptional regulation, and are critical in normal development and genetic diseases, but we lack effective rules to predict their function from sequence alone. Taking advantage of the abundant and optically clear embryos and rapid early development of the zebrafish, we have developed a transposon-based system to evaluate the function of potential enhancer elements. We are applying this approach to key developmental genes, to identify enhancers acting in bone and cartilage, dopaminergic neurons, pancreatic islets, and other cell types. Identification of these enhancers is allowing us to unravel the signaling pathways controlling normal development of critical tissues, and ultimately to manipulate those pathways in culture to direct stem cell differentiation.

Cell Research (2008) 18:s20. doi: 10.1038/cr.2008.110; published online 4 August 2008

Correspondence: Shannon Fisher

Shannon Fisher, $\mathrm{MD}, \mathrm{PhD}$, received from her $\mathrm{MD}$ and $\mathrm{PhD}$ degrees from Johns Hopkins University School Medicine (JHUSOM) in 1993 and completed postdoctoral training in Carnegie Institute of Embryology. She took an Assistant Professor in JHUSOM in 1999. She is currently faculty member of Institute for Genetic Medicine and in Departments of Cell
Biology and Medicine. Dr Fisher laboratory is currently focusing on using the zebrafish as a model system for studying genetics and developmental biology, esp. skeletal development. Their recent contributions include the development of new reagents and technologies for the study of skeletogenesis in the zebrafish. See more details: www.hopkinsmedicine. org/geneticmedicine/People/Faculty/fisher.html 